\title{
Evaluation of a petit tissue microarray in a tertiary care histopathology laboratory- a prospective study
}

\author{
Prasaad P.R. ${ }^{1}$, Srilakshmi Priya V. ${ }^{2}$, Rao B.G ${ }^{3}$, Parijatham B.O. ${ }^{4}$, Lakshmi Prabha.V. ${ }^{5}$ \\ ${ }^{1}$ Dr. Priavadhana Rajan Prasaad, Assistant Professor, affiliated to ESIC Medical College and PGIMSR, KK Nagar, \\ Chennai-78, ${ }^{2}$ Dr. Srilakshmi Priya V, Post Graduate Student, ${ }^{3}$ Dr. Bheema Rao G., Associate Professor, ${ }^{4}$ Dr. B.O. \\ Parijatham, Professor, ${ }^{5}$ Dr. Lakshmi Prabha V, Senior Lab Technician, all authors are affiliated with Department of \\ Pathology, Sree Balaji Medical College and Hospital, Chromepet, Chennai, Tamilnadu, India.
}

Corresponding Author: Dr. Priavadhana Rajan Prasaad, Assistant Professor, ESIC Medical College and PGIMSR, KK Nagar, Chennai-78, Email: drpriarajan@gmail.com

\begin{abstract}
Background: With the advancements in pathology for diagnosis of tumors, there is a need for technologies which provide test results with a short turn-around time. Also, with the increasing incidence of early diagnosis of tumor, detection of prognostic markers becomes a necessity. Tissue Micro-Array is one such technology in which tumor diagnosis, tumor markers and prognostic markers could be studied with limited tissue sample at a low cost. In this study, we evaluated the feasibility of a petit Tissue Microarray for immunohistochemical profiling of breast carcinomas. Materials and Methods: Tissue cores were obtained from random tissues which included placenta, breast tissue and lymphnode and endometrium using skin punch biopsy needle of bore $2 \mathrm{~mm}$. These were done for standardizing the procedure of a miniature tissue microarray. Further, tissue cores obtained from carcinoma breast tissue by two different methods were used for constructing a microarray block. Sections of 4 micron thickness were taken and stained with hematoxylin and eosin stains. If satisfactory tumor tissue is present in this constructed block, then sections were taken for immunohistochemistry staining with ER, PR and HER2. Results: Directly constructed tissue blocks had better preservation of tumor tissue morphology compared to the blocks constructed from donor blocks. Also, directly constructed tissue blocks had the advantage of not mutilating the donor block which could be still used for further studies and reference. Immunohistochemistry revealed similar results as obtained during the routine histopathological sections. Also, the cost of the reagents used for immunohistochemistry was reduced by $200 \%$ as compared to the routine immunohistochemical staining procedure. Conclusion: A petit Tissue microarray is definitely possible in a tertiary care histopathology laboratory and can be utilized for immunohistochemical studies with multiple markers.
\end{abstract}

Keywords: Microarray, Core Biopsy, Skin punches, Donor blocks

\section{Background}

With the advancements in pathology for diagnosis of tumors, there is a need for technologies which provide test results with a short turn-around time.Also, with the increasing incidence of early diagnosis of tumor, detection of prognostic markers becomes a necessity. Tissue Micro-Array (TMA) is one such technology in which tumor diagnosis, tumor markers and prognostic markers could be studied with limited tissue sample at a low cost $[1,2]$.

Initially designed for basic research, TMA technology has currently gained importance in the field of cancer research [3]. Multiple Gene analysis in patients with

Manuscript received: $30^{\text {th }}$ July 2018

Reviewed: $8^{\text {th }}$ August 2018

Author Corrected: $14^{\text {th }}$ August 2018

Accepted for Publication: $18^{\text {th }}$ August 2018 high risk factors is possible with this microarray technology. Construction of TMA blocks can be done either manually or by automated machines. In high volume centres, automated technology is feasible.

But in low volume centres with small sample size and minimal tissue availability, manual construction becomes mandatory.

Yet, construction of microarray blocks manually is a labour-intensive process and also time consuming.

Hence, in this study, we evaluated the feasibility of a petit Tissue Microarray (a small TMA block) for immunohistochemical profiling of breast carcinomas. 


\section{Original Research Article}

\section{Materials and Methods}

This was a prospective study conducted in the Department of Pathology during the period of July 2016-

December 2016. After the routine identification and grossing of specimens, mastectomy specimens with tumor masses were selected for constructing the Tissue microarray blocks.

Inclusion criteria: All mastectomy specimens with grossly appreciable tumor masses were selected for construction of petit TMA blocks

Exclusion criteria: Mastectomy specimens post therapy, with no grossly appreciable tumor were excluded for constructing blocks

These petit Tissue Micro Array ( $p$ TMA) blocks were constructed by two different methodologies. Skin biopsy punches with a diameter of $2 \mathrm{~mm}$ bore were used for sampling.

\section{Method-1: Classical Indirect Method CIM)}

In this method, the classical manual construction of a TMA block was followed.First a donor block with appropriate tumor tissue was selected and their corresponding histopathology slide studied. Then, the area of maximal tumor tissue was marked in the block and using a skin biopsy punch, tissue cores were bored in the donor block and transferred to the recipient block. Multiple sites were marked in the donor block and punches made to avoid sampling error and to compensate for losses during section processing. 4 cores were made from each specimen and routinely processed for immunohistochemical staining.

\section{Method-2: Modified Direct Method (MDM)}

Mastectomy specimens are routinely grossed and bits taken for histopathological reporting. After routine grossing, skin biopsy punches are used to sample tissues directly from the tumor site. Multiple punches are made to minimize sampling error.

These are fixed in 10\% neutral buffered formalin and routinely processed. While making the paraffin block with Leukart's L pieces, 4 tissue cores from each specimen was transferred to a single block to construct a miniature TMA block.Sections were cut with 4 micron thickness and routinely processed for immunohistochemical staining.

Standardisation of Method 2 (MDM): The technique of constructing a direct TMA from the tissue itself has to be standardized before processing for immunohistochemistry for cancer profiling. Hence, we selected random tissue samples which included placenta FIG 3, endometrium FIG 4, lymphnode FIG 5 and normal breast tissue FIG6 for construction of a TMA block and the results were evaluated before designing of TMA for mastectomy tumor specimens.

After construction of $p$ TMA blocks by the above two methods, 4 micron sections were taken and hematoxylin - eosin staining done. These slides were evaluated for the representative tissue sampling and then processed for Immunohistochemistry of Estrogen receptors, Progesterone receptors and Her 2 expression. The slides were then studied by pathologist for the presence of appropriate representation of material and the two different methods of construction of blocks were compared.

\section{Results}

We designed five petit TMA blocks from each of the methods above mentioned i.e. and the classical indirect method FIG 1 and modified direct method FIG 2.
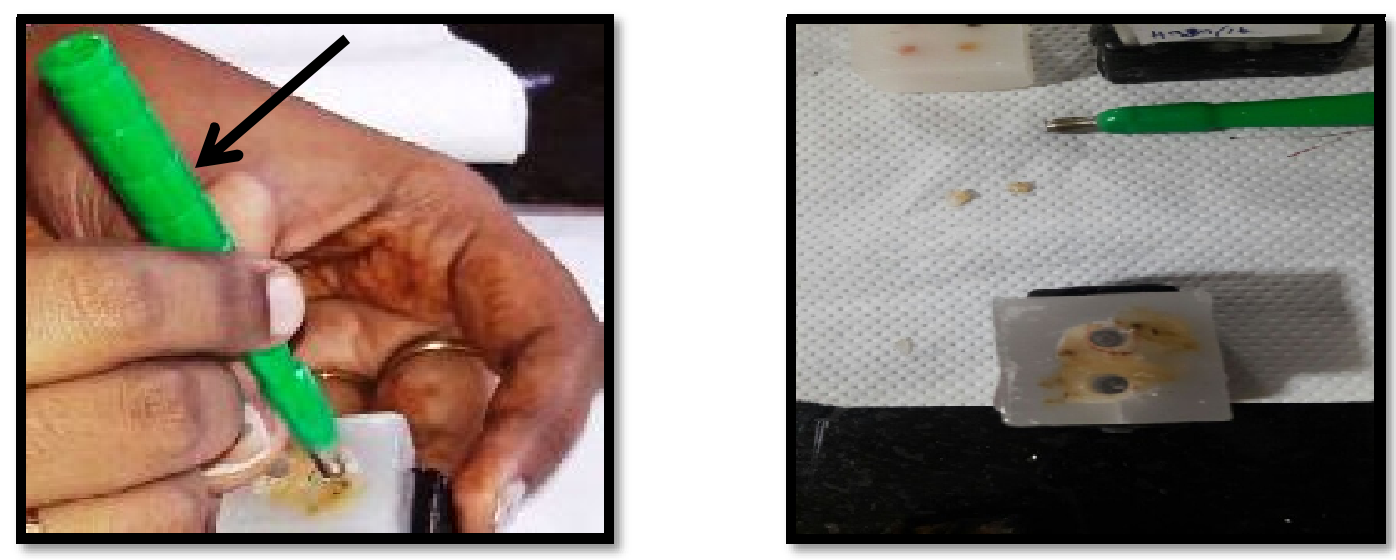

Fig-1: Classical Indirect Method 


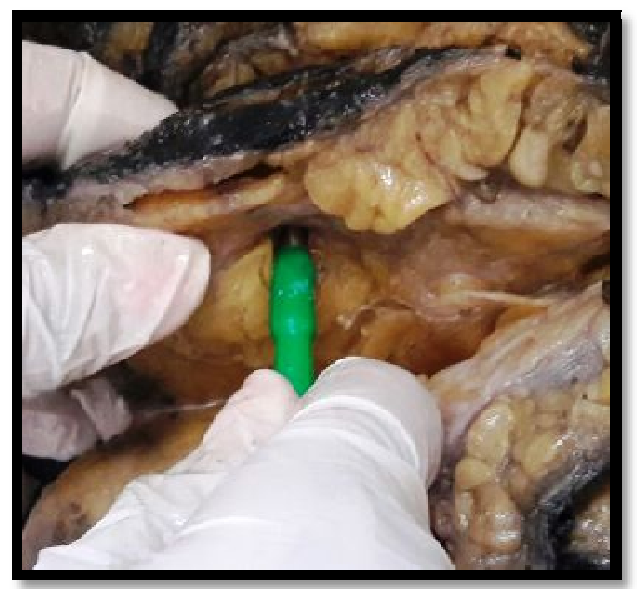

\section{Original Research Article}

Fig-2: Modified Direct Method

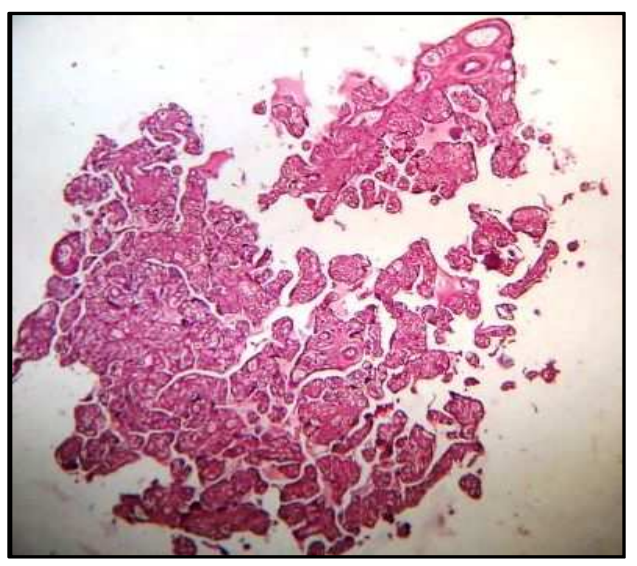

Fig- 3: Control Placenta (MDM)

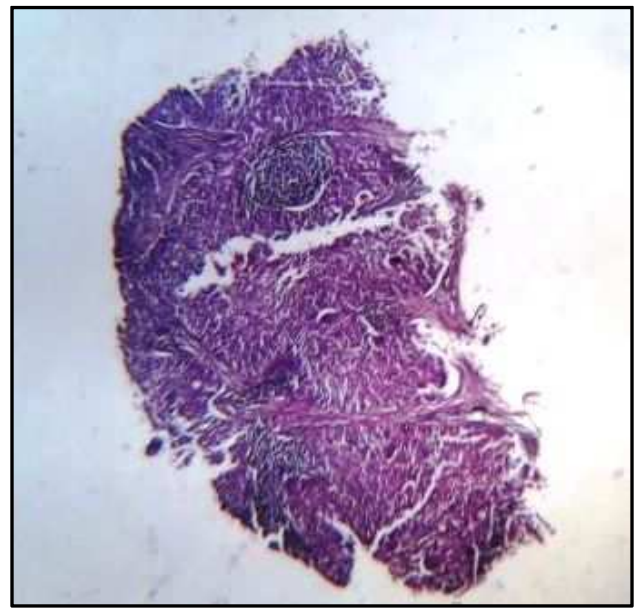

Fig-5: Control Lymph Node (MDM)

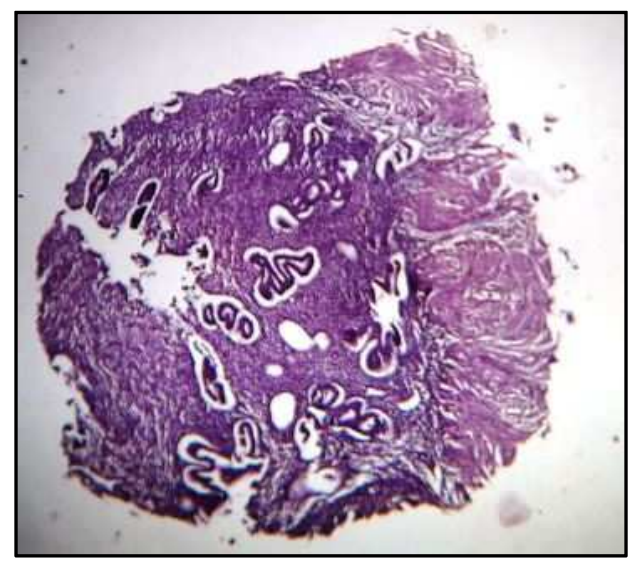

Fig-4: Control Endometrium (MDM)

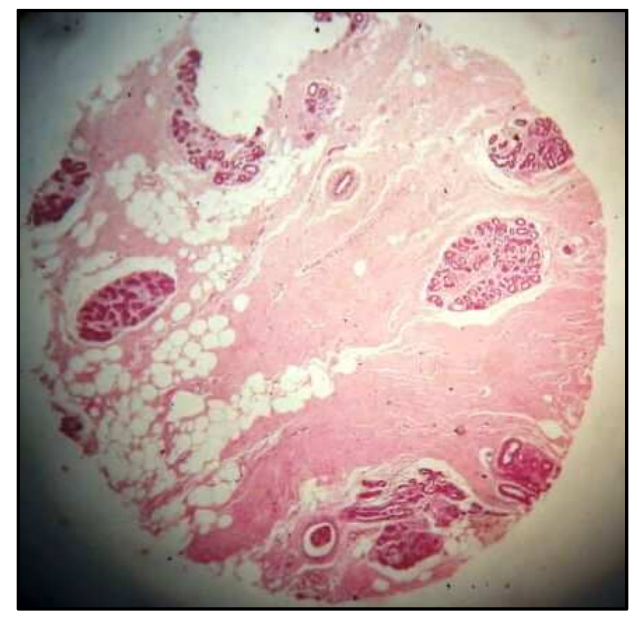

Fig-6: Control Normal B reast (MDM)

Total time required for construction of these $p$ TMA was 30 minutes (Blocks with 4 cores only) both by the direct and the indirect methods.

During section cutting, defects like loss of tissue was noted in one of the five blocks $(20 \%)$ constructed using the indirect method and no washing out of tissue noted in the direct method.

H\& E staining was found to be satisfactory in both the methods. Subsequent immunohistochemical detection of estrogen and progesterone receptors were satisfactory and comparable to the routine blocks. There were sampling defects noted in one of the five blocks (20\%) constructed using the direct method and none noted in the indirect method. 


\section{Original Research Article}

\section{Discussion}

In this era of rapidly advancing technology, there is a need for rapid results and multiple marker evaluation. Tissue Microarray was first developed by Dr. Hector Battifora with tissue cores from different tissues for a particular antigen expression [1,2]. Modifications on this basic invention lead on to the current method of arraying technology. This arraying technology has revolutionized over the past years and has been applied for methods like comparative genomic hybridization, cDNA detection and next generation sequencing. The major advantage of TMA is that a large number of patient samples can be analyzed for different studies in a very cost-effective way. But, use of this technique is time consuming and also less traceable to the patient. Also, a major disadvantage of this technique is the sampling error since only a small fragment of tissue is subjected to studies like histochemistry, immunohistochemistry or in-situ hybridization.

There are various types of TMAs available like Cell line Arrays, Random tumor Arrays, Consecutive case array, Tumor characteristic based Array etc. Also, TMA blocks could be constructed by manual tissue arraying technique or using automated tissue arrayers [2].

The major applications of these TMAs are for validation of complementary DNA analysis, validating the sensitivity and specificity of a new antibody, for quality assurance in histopathology and immunohistochemistry. Singh et alin their review on TMA have discussed about the spectrum of applications of TMA [3]. They have mentioned in their review, that the major applications of TMA are for validation of diagnostic biomarkers, validation of prognostic biomarkers, evaluation of clinical response to therapy, research in neurodegenerative diseases. Also, TMAs are being increasingly used for quality control in immunohistochemistry. New emerging modified TMAs are frozen TMAs, Cell line Microarrays, Xenograft tumor assays and Tissue immunoblotting. They have also discussed that the limitations of TMA are the inadequate representation of tumor tissues and due to differential expression of tumor antigens in different parts of the tumor (due to tumor heterogeneity) [7]. There is a vast variety of automated tissue microarrayers available commercially. Beecher instruments, Viridian and Unitma are some of the manufacturers who provide TMA machines. Yet, due to the high cost of these manual as well as the automated tissue microarrayers, centres with low volume of pathological samples, cannot afford to the high cost and maintenance of these instruments.Hence, there is a need for design of TMA blocks with simple and easily available equipment. In our study, we evaluated the feasibility of TMA construction using a skin biopsy punch.

Choi $\mathrm{CH}$ et al in their study, have evaluated the possibility of construction of high-density TMAs at low costs using selfmade manual microarray kits [4]. In this study, they have constructed a TMA using ordinary cannula piercing needles, metallic ink cartridges of ballpoint pens, skin biopsy punches and bone marrow biopsy needles and have shown that they could design a high-density TMA using these inexpensive simple tools. Yet, they have also stated that, in the process of this designing, they have observed that there are chances of cross-contamination of tissues and loss of tissue cores during section cutting and processing.

One of the advantages of our method of Modified direct way of construction of TMAs, is that, there is no residual paraffin from the donor block and hence, the chances of cross-contamination of tissues is greatly minimized. Also, reusability of the skin punches or the instruments used for making tissue cores is high with direct modified method rather than the donor-recipient indirect method of TMA construction. This is due to the presence of residual wax in the tips of skin punches observed during the indirect classical method.

It was observed in this study, that while construction of blocks by the classical indirect method using the donor and recipient blocks, there was total loss of the donor block which could not be further used for any studies. In contrast, in the modified direct methods of petit TMA construction, it was observed that, since these were made from direct tissue samples, there is always a possibility of using the routine histopathology blocks for further studies. Table $\mathbf{1}$ enumerates the advantages and disadvantages of the different methods employed to construct tissue microarray in this study.

Table -1

\begin{tabular}{|c|c|c|}
\hline Features & MDM (Modified direct method) & CIM (Classical indirect method) \\
\hline Donor block reuse & Possible & Not possible \\
\hline Sampling defect & Occurs in $20 \%$ of cases & Rarely seen \\
\hline Loss of tissue during processing & Rarely seen & Occurs in $20 \%$ of cases \\
\hline
\end{tabular}




\section{Original Research Article}

During routine immunohistochemistry, control blocks are run along with the pathological samples for study in a separate slide. But, with this $p$ TMA procedure, since control is also run in the same block and in a single slide, there are minimal chances of variation in the results and these controls are also easily comparable with the pathological sample results $[6,7,8,9]$.

Shebl et al in their study, have validated an inexpensive method of small paraffin microarrays using mechanical pencil tips [5]. In their study, they have manually constructed tissue microarray blocks using mechanical pencil tips of $1 \mathrm{~mm}$ diameter. They have concluded that the time was greatly reduced and only little tissue damage to the donor blocks since the diameter of pencil tip was very small.Also, they have concluded in their study, immunodetection could also be successfully performed using this technique.

Table-2: Comparison of the different methods used for constructing manual TMA blocks.

\begin{tabular}{|c|c|c|c|}
\hline SL. No & Study by & Method & Bore size \\
\hline 1. & Shebl et al & Mechanical pencil tips & $1 \mathrm{~mm}$ \\
\hline 2. & Choi H et al & Metallic ink cartridges of ballpoint pens & $0.6 \mathrm{~mm}-2 \mathrm{~mm}$ \\
\hline 3. & Singh et al & $11-19$ G bone marrow needle & $1-3 \mathrm{~mm}$ \\
\hline 4. & Our study & Skin biopsy punches & $2 \mathrm{~mm}$ \\
\hline
\end{tabular}

Chavan SS et al in their study on 53 cases of breast carcinoma, compared the utility automated TMA cores and whole sections for the immunohistochemical expression of ER, PR and HER2 [10]. In their study, they have observed that significant concordance rates are found between the blocks constructed using whole sections and those constructed using automated tissue microarrayer. This is similar to our study, in which we have studied the expression of breast markers in both whole sections and manually constructed petit TMA blocks and found that both showed similar degrees of expression of the receptors.

Srinath $\mathrm{S}$ et al in their study evaluated the utility of manual construction of TMA blocks using a wax mould, silicone mould and compared it with an automated tissue microarrayer blocks [11].

They have observed that silicone moulds were a cheap alternative as well as could be standardised easily compared to the wax moulds. In our study, we constructed the recipient block by the tissues obtained directly from the specimens in the modified direct method. In this way, it will be similar to a routine histopathology whole block section except that due to the thin size of the cores, the different cores could be placed at different levels in the recipient block. This may lead to sectioning defects or lead to non-representative sections.

Bhargava $\mathrm{R}$ et al in their study, compared the expression of HER2 by FISH in TMA blocks and by IHC in whole tissue sections on 114 invasive breast carcinomas [12]. They have observed that HER2 detected by FISH had a 99\% concordance rate with HER2 detected by IHC in whole tissue sections. They have concluded not only IHC could be performed in TMA blocks effectively, but FISH could be performed with reliable results.

\section{Conclusion}

A petit Tissue microarray is definitely possible in a tertiary care histopathology laboratory and can be utilized for immunohistochemical studies with multiple markers.

Tissue microarray performed using automated techniques are expensive and unaffordable in a tertiary care laboratory where the volume of cases is less compared to the cost involved in the technique.

Hence, manual construction of a petit Tissue microarray as performed in this study, could be an inexpensive alternative to automated Tissue microarrayers.
Contributions: The paper was written in collaboration with all the authors. PRP and BRG defined the research idea. All data collection and techniques involved were carried out by SPV and LPV. Analysis of data was carried out by PRP and SPV. PRP drafted the paper and $\mathrm{BOP}$ and BRG reviewed and finalised the manuscript

Funding: Nil, Conflict of interest: None initiated Permission from IRB: Yes

\section{References}

1. Bancroft J. Histopathological stains and their diagnostic uses. Edinburgh: Churchill Livingstone; 1975. 


\section{Original Research Article}

2. Kallioniemi OP, Wagner U, Kononen J, Sauter G. Tissue microarray technology for high-through put molecular profiling of cancer. Hum Mol Genet. 2001 Apr; 10(7):657-62.

3. Avninder S, Ylaya K, Hewitt S. Tissue microarray: A simple technology that has revolutionized research in pathology. Journal of Postgraduate Medicine. 2008; 54 (2):158.doi.10.4103/0022-3859.40790

4. Choi C, Kim K, Song J, Choi S, Kim L, Park I et al. Construction of High-Density Tissue Microarrays at Low Cost by Using Self-Made Manual Microarray Kits and Recipient Paraffin Blocks. Korean Journal of Pathology. 2012;46(6):562.doi.10.4132/KoreanJ Pathol. 2012.46.6.562

5. Shebl A, Zalata K, Amin M, El-Hawary A. An inexpensive method of small paraffin tissue microarrays using mechanical pencil tips. Diagn Pathol. 2011;6 (1): 117.doi/10.1186/1746-1596-6-1176.

6. Tripodi SA, Rocca BJ, Hako L, Barbagli L, Bartolommei S, Ambrosio MR. Quality control by tissue microarray in immunohistochemistry. $\mathbf{J}$ Clin Pathol. 2012; Jul; 65 (7): 635-7. doi: 10.1136/jclinpath2011-200551.

7. Gulbahce H, Gamez R, Dvorak L, Forster C, Varghese L. Concordance Between Tissue Microarray and Whole-section Estrogen Receptor Expression and Intratumoral Heterogeneity. ApplImmunohistochemi
MolMorphol. 2012;20(4):340-343.doi: 10.1097/PAI. 0b013e 318241ca14

8. Mengel M, Kreipe H, von Wasielewski R. Rapid and Large-Scale Transition of New Tumor Biomarkers to Clinical Biopsy Material by Innovative Tissue Microarray Systems. ApplImmunohistochem Molr Morpho1. 2003;11(3)261-268.

9. Matysiak B, Brodzeller T, Buck S, French A, Counts C, Boorsma B et al. Simple, Inexpensive Method for Automating Tissue Microarray Production Provides Enhanced Microarray Reproducibility. Appl Immunohistochem Mol Morphol. 2003;11(3)269-273.

10. Chavan SS, Ravindra S, Prasad M. Breast Biomarkers-Comparison on Whole Section and Tissue Microarray Section. J Clin Diagn Res :JCDR. 2017; 11(3): EC40-EC44. doi:10.7860/JCDR/2017/ 25088. 9573.

11. Srinath S, Kendole RK, Gopinath P, Krishnappa S, Vishwanath S. Economic methods used in fabrication of tissue microarray: A pilot study. J Oral Maxillofac Pathol : JOMFP. 2016;20(1):86-90. doi:10.4103/0973029X.180948.

12. Bhargava R, Lal P, Chen B. Feasibility of using tissue microarrays for the assessment of HER-2 gene amplification by fluorescence in situ hybridization in breast carcinoma. Diagn Mol Pathol. 2004 Dec;13(4): 213-6.

\section{How to cite this article?}

Prasaad P.R, Srilakshmi Priya V, Rao B.G, Parijatham B.O, Lakshmi Prabha.V. Evaluation of a petit tissue microarray in a tertiary care histopathology laboratory- a prospective study. Trop J Path Micro 2018; 4(4):353-358.doi:10. 17511/ jopm. 2018.i4.10 